\title{
A GENERALIZATION \\ OF THE CLASSIC COMBINATION RULES TO DSm HYPER-POWER SETS
}

\begin{abstract}
Milan DANIEL
Abstract: Dempster's rule, Yager's rule and Dubois-Prade's rule for belief functions combination are generalized to be applicable to hyper-power sets according to the DSm theory. A comparison of the rules with DSm rule of combination is presented.
\end{abstract}

Keywords: Dempster-Shafer theory, Dempster's rule, Yager's rule, Dubois-Prade's rule, DSm theory, hyper-power set, DSm model, DSm rule of combination.

\section{Introduction}

Belief functions are one of the widely used formalisms for uncertainty representation and processing. Belief functions enable representation of incomplete and uncertain knowledge, belief updating and combination of evidence. Belief functions were originally introduced as a principal notion of Dempster-Shafer Theory (DST) or the Mathematical Theory of Evidence [12].

For a combination of beliefs Dempster's rule of combination is used in DST. Under strict probabilistic assumptions, its results are correct and probabilistically interpretable for any couple of belief functions. Nevertheless these assumptions are rarely fulfilled in real applications. It is not uncommon to find examples where the assumptions are not fulfilled and where results of Dempster's rule are counter-intuitive, e.g. see $[1,2,13]$, thus a rule with more intuitive results is required in such situations.

Hence, a series of modifications of Dempster's rule were suggested and alternative approaches were created. The classical ones are Dubois and Prade's rule [9] and Yager's belief combination rule [15]. Others include a wide class of weighted operators [11], the Transferable Belief Model (TBM) using the so-called non-normalized Dempster's rule [14], disjunctive (or dual Demspter's) rule of combination [4, 8], combination 'per elements' with its special case - minC combination, see [3,5], and other combination rules. It is also necessary to mention the method for application of Dempster's rule in the case of partially reliable input beliefs [10].

INFORMATION \& SECURITY. An International Journal, Vol. 20, 2006, 46-57 
A brand new approach performs the Dezert-Smarandache (or Demspter-Shafer modified) theory (DSmT) with its DSm rule of combination. There are two main differences: 1) mutual exclusivity of elements of a frame of discernment is not assumed in general; mathematically it means that belief functions are not defined on the power set of the frame, but on a so-called hyper-power set, i.e., on the Dedekind lattice defined by the frame; 2) a new combination mechanism which overcomes problems with conflict among the combined beliefs and which also enables a dynamic fusion of beliefs.

As the classical Shafer's frame of discernment may be considered the special case of a so-called hybrid DSm model, the DSm rule of combination is compared with the classic rules of combination in the publications about DSmT [7, 13].

Unfortunately, none of the classical combination rules has been formally generalized to hyper-power sets, thus their comparison with the DSm rule is not fully objective until now.

The present paper brings a formal generalization of the classical Dempster's, DuboisPrade's, and Yager's rules to hyper-power sets. These generalizations perform a solid theoretical background for a serious objective comparison of the DSm rule with the classical combination rules.

The classic definitions of Dempster's, Dubois-Prade's, and Yager's combination rules are briefly recalled in Section 2 and basic notions of DSmT (Dedekind lattice, hyper-power set, DSm models, and DSm rule of belief combination) in Section 3.

A generalization of Dempster's rule is presented in Section 4, and a generalization of Yager's rule in Section 5. Both these classic rules are straightforwardly generalized as their ideas work on hyper-power sets simply without any problem.

More interesting and more complicated is the case of Dubois-Prade's rule. The nature of this rule is closer to DSm rule, but on the other hand the generalized DuboisPrade's rule is not compatible with a dynamic fusion in general. It works only for a dynamic fusion without non-existential constraints, whereas a further extension of the generalized rule is necessary in the case of a dynamic fusion with non-existential constraints.

Section 7 presents a brief comparison of the rules and open problems for a future research. A concluding section follows.

\section{Classic definitions}

All the classic definitions assume an exhaustive finite frame of discernment $\Theta=$ $\left\{\theta_{1}, \ldots, \theta_{n}\right\}$, whose elements are mutually exclusive.

A basic belief assignment (bba) is a mapping $m: \mathcal{P}(\Theta) \longrightarrow[0,1]$, such that $\sum_{A \subset \Theta} m(A)=1$, the values of bba are called basic belief masses $(\mathrm{bbm})$. The value $m(A)$ is called the basic belief mass $(b b m)$ of $A .{ }^{1}$ A belief function $(B F)$ is a mapping

\footnotetext{
${ }^{1} m(\emptyset)=0$ is often assumed in accordance with Shafer's definition [12]. A classic counter example is
} 
Bel $: \mathcal{P}(\Theta) \longrightarrow[0,1], \operatorname{bel}(A)=\sum_{\emptyset \neq X \subseteq A} m(X)$, belief function Bel uniquely corresponds to bba $m$ and vice-versa. $\mathcal{P}(\Theta)$ is often denoted also by $2^{\Theta}$. A focal element is a subset $X$ of the frame of discernment $\Theta$, such that $m(X)>0$. If a focal element is a one-element subset of $\Theta$, we are referring to a singleton.

Let us start with the classic definition of Dempster's rule. Dempster's (conjunctive) rule of combination $\oplus$ is given as

$\left(m_{1} \oplus m_{2}\right)(A)=\sum_{X, Y \subseteq \Theta, X \cap Y=A} K m_{1}(X) m_{2}(Y)$ for $A \neq \emptyset$, where $K=\frac{1}{1-\kappa}$, $\kappa=\sum_{X, Y \subset \Theta, X \cap Y=\emptyset} m_{1}(X) m_{2}(Y)$, and $\left(m_{1} \oplus m_{2}\right)(\emptyset)=0$, see [12]; putting $K=1$ and $\left(m_{1} \oplus m_{2}\right)(\emptyset)=\kappa$ we obtain the non-normalized conjunctive rule of combination $\odot$, see e. g. [14].

Yager's rule of combination (B), see [15], is given as $\left(m_{1}\left(m_{2}\right)(A)=\sum_{X, Y \subset \Theta, X \cap Y=A} m_{1}(X) m_{2}(Y)\right.$ for $\emptyset \neq A \subset \Theta$, $\left(m_{1}\left(m_{2}\right)(\Theta)=m_{1}(\Theta) m_{2}(\Theta)+\sum_{X, Y \subseteq \Theta, X \cap Y=\emptyset} m_{1}(X) m_{2}(Y)\right.$, and $\left(m_{1} \otimes m_{2}\right)(\emptyset)=0$;

Dubois-Prade's rule of combination $\circledast$ is given as $\left(m_{1} \circledast m_{2}\right)(A)=$ $\sum_{X, Y \subseteq \Theta, X \cap Y=A} m_{1}(X) m_{2}(Y)+\sum_{X, Y \subseteq \Theta, X \cap Y=\emptyset, X \cup Y=A} m_{1}(X) m_{2}(Y)$ for $\emptyset \neq A \subseteq \Theta$, and $\left(m_{1} \circledast m_{2}\right)(\emptyset)=0$, see [9].

\section{Introduction to the DSm theory}

Because DSmT is a new theory which is in permanent dynamic evolution, we have to note that this text is related to its state described by formulas and text presented in the basic publication on DSmT - in the book [13]. Rapid development of the theory is demonstrated by announcement of the second book on DSmT.

\subsection{Dedekind lattice, basic DSm notions}

Dempster-Shafer modified Theory or Dezert-Smarandache Theory (DSmT) by Dezert and Smarandache [7, 13] allows mutually overlapping elements of a frame of discernment. Thus, a frame of discernment is a finite exhaustive set of elements $\Theta=\left\{\theta_{1}, \theta_{2}, \ldots, \theta_{n}\right\}$, but not necessarily exclusive in DSmT. As an example, we can introduce a three-element set of colours $\{$ Red, Green, Blue $\}$ from the DSmT homepage $^{2}$. DSmT allows that an object can have 2 or 3 colours at the same time: e.g. it can be both red and blue, or red and green and blue in the same time, it corresponds to a composition of the colours from the 3 basic ones.

DSmT uses basic belief assignments and belief functions defined analogically to the classic Dempster-Shafer theory (DST), but they are defined on a so-called hyperpower set or Dedekind lattice instead of the classic power set of the frame of discern-

Smets' Transferable belief model (TBM) which admits positive $m(\emptyset)$ as it assumes $m(\emptyset) \geq 0$.

${ }^{2}$ www.gallup.unm.edu/ smarandache/DSmT.htm 
ment. To be distinguished from the classic definitions, they are called generalized basic belief assignments and generalized basic belief functions.

The Dedekind lattice, more frequently called hyper-power set $D^{\Theta}$ in DSmT, is defined as the set of all composite propositions built from elements of $\Theta$ with union and intersection operators $\cup$ and $\cap$ such that $\emptyset, \theta_{1}, \theta_{2}, \ldots, \theta_{n} \in D^{\Theta}$, and if $A, B \in D^{\Theta}$ then also $A \cup B \in D^{\Theta}$ and $A \cap B \in D^{\Theta}$, no other elements belong to $D^{\Theta}\left(\theta_{i} \cap \theta_{j} \neq \emptyset\right.$ in general, $\theta_{i} \cap \theta_{j}=\emptyset$ iff $\theta_{i}=\emptyset$ or $\theta_{j}=\emptyset$ ).

Thus the hyper-power set $D^{\Theta}$ of $\Theta$ is closed to $\cup$ and $\cap$ and $\theta_{i} \cap \theta_{j} \neq \emptyset$ in general. Whereas the classic power set $2^{\Theta}$ of $\Theta$ is closed to $\cup, \cap$ and complement, and $\theta_{i} \cap \theta_{j}=\emptyset$ for every $i \neq j$.

Examples of hyper-power sets. Let $\Theta=\left\{\theta_{1}, \theta_{2}\right\}$, we have $D^{\Theta}=\left\{\emptyset, \theta_{1} \cap\right.$ $\left.\theta_{2}, \theta_{1}, \theta_{2}, \theta_{1} \cup \theta_{2}\right\}$, i.e. $\left|D^{\Theta}\right|=5$. Let $\Theta=\left\{\theta_{1}, \theta_{2}, \theta_{3}\right\}$ now, we have $D^{\Theta}=$ $\left\{\alpha_{0}, \alpha_{1}, \ldots \alpha_{18}\right\}$, where $\alpha_{0}=\emptyset, \alpha_{1}=\theta_{1} \cap \theta_{2} \cap \theta_{3}, \alpha_{2}=\theta_{1} \cap \theta_{2}, \alpha_{3}=\theta_{1} \cap \theta_{3}, \ldots, \alpha_{17}=$ $\theta_{2} \cup \theta_{3}, \alpha_{18}=\theta_{1} \cup \theta_{2} \cup \theta_{3}$, i.e., $\left|D^{\Theta}\right|=19$ for $|\Theta|=3$.

A generalized basic belief assignment $(\mathrm{gbba}) m$ is a mapping $m: D^{\Theta} \longrightarrow[0,1]$, such that $\sum_{A \in D^{\Theta}} m(A)=1$ and $m(\emptyset)=0$. The quantity $m(A)$ is called the generalized basic belief mass $(\mathrm{gbbm})$ of $A$. A generalized belief function $(\mathrm{gBF}) \mathrm{Bel}$ is a mapping $B e l: D^{\Theta} \longrightarrow[0,1]$, such that $\operatorname{Bel}(A)=\sum_{X \subset A, X \in D^{\Theta}} m(X)$, generalized belief function $\mathrm{Bel}$ uniquely corresponds to gbba $m$ and vice-versa.

\subsection{DSm models}

If we assume a Dedekind lattice (hyper-power set) according to the above definition without any other assumptions, i.e., all elements of an exhaustive frame of discernment can mutually overlap themselves, we refer to the free $\operatorname{DSm}$ model $\mathcal{M}^{f}(\Theta)$, i.e., about the DSm model free of constraints.

In general it is possible to add exclusivity or non-existential constraints into DSm models, we speak about hybrid DSm models in such cases.

An exclusivity constraint $\theta_{1} \cap \theta_{2} \stackrel{\mathcal{M}_{1}}{\equiv} \emptyset$ says that elements $\theta_{1}$ and $\theta_{2}$ are mutually exclusive in model $\mathcal{M}_{1}$, whereas both of them can overlap with $\theta_{3}$. If we assume exclusivity constraints $\theta_{1} \cap \theta_{2} \stackrel{\mathcal{M}_{2}}{\equiv} \emptyset, \theta_{1} \cap \theta_{3} \stackrel{\mathcal{M}_{2}}{\equiv} \emptyset, \theta_{2} \cap \theta_{3} \stackrel{\mathcal{M}_{2}}{=} \emptyset$, another exclusivity constraint directly follows them: $\theta_{1} \cap \theta_{2} \cap \theta_{3} \stackrel{\mathcal{M}_{2}}{=} \emptyset$. In this case all the elements of the 3-element frame of discernment $\Theta=\left\{\theta_{1}, \theta_{2}, \theta_{3}\right\}$ are mutually exclusive as in the classic Dempster-Shafer theory, and we call such hybrid DSm model as Shafer's model $\mathcal{M}^{0}(\Theta)$.

A non-existential constraint $\theta_{3} \stackrel{\mathcal{M}_{3}}{=} \emptyset$ brings additional information about a frame of discernment saying that $\theta_{3}$ is impossible; it forces all the gbbm of $X \subseteq \theta_{3}$ to be equal to zero for any gbba in model $\mathcal{M}_{3}$. It represents a sure meta-information with respect to generalized belief combination which is used in a dynamic fusion.

In a degenerated case of the degenerated DSm model $\mathcal{M}_{\emptyset}$ we always have $m(\emptyset)=$ $1, m(X)=0$ for $X \neq \emptyset$. It is the only case where $m(\emptyset)>0$ is allowed in DSmT. 
The total ignorance on $\Theta$ is the union $I_{t}=\theta_{1} \cup \theta_{2} \cup \ldots \cup \theta_{n} . \emptyset=\left\{\emptyset_{\mathcal{M}}, \emptyset\right\}$, where $\emptyset_{\mathcal{M}}$ is the set of all elements of $D^{\Theta}$ which are forced to be empty through the constraints of the model $\mathcal{M}$ and $\emptyset$ is the classic empty set ${ }^{3}$. For a given DSm model we can define (in addition to [13]) $\Theta_{\mathcal{M}}=\left\{\theta_{i} \mid \theta_{i} \in \Theta, \theta_{i} \notin \emptyset_{\mathcal{M}}\right\}, \Theta_{\mathcal{M}} \stackrel{\mathcal{M}}{\equiv} \Theta$, and $I_{\mathcal{M}}=\bigcup_{\theta_{i} \in \Theta_{\mathcal{M}}} \theta_{i}$, i.e. $I_{\mathcal{M}} \stackrel{\mathcal{M}}{\equiv} I_{t}, I_{\mathcal{M}}=I_{t} \cap \Theta_{\mathcal{M}}, I_{\mathcal{M}_{\emptyset}}=\emptyset$.

\subsection{The DSm rule of combination}

The classic DSm rule DSmC is defined on the free DSm models as it follows ${ }^{4}$ : $m_{\mathcal{M}^{f}(\Theta)}(A)=\left(m_{1} \circledast m_{2}\right)(A)=\sum_{X, Y \in D^{\Theta}, X \cap Y=A} m_{1}(X) m_{2}(Y)$.

Since $D^{\Theta}$ is closed under operators $\cap$ and $\cup$ and all the $\cap$ s are non-empty, the classic DSm rule guarantees that $\left(m_{1} \circledast m_{2}\right)$ is a proper generalized basic belief assignment. The rule is commutative and associative. For n-ary version of the rule see [13].

When the free DSm model $\mathcal{M}^{f}(\Theta)$ does not hold due to the nature of the problem under consideration, which requires us to take into account some known integrity constraints, one has to work with a proper hybrid DSm model $\mathcal{M}(\Theta) \neq \mathcal{M}^{f}(\Theta)$. In such a case, the hybrid DSm rule of combination $D S m H$ based on the hybrid model $\mathcal{M}(\Theta)$, $\mathcal{M}^{f}(\Theta) \neq \mathcal{M}(\Theta) \neq \mathcal{M}_{\emptyset}(\Theta)$, for $k \geq 2$ independent sources of information is defined as: $m_{\mathcal{M}(\Theta)}(A)=\left(m_{1} \circledast m_{2} \circledast \ldots \circledast m_{k}\right)(A)=\phi(A)\left[S_{1}(A)+S_{2}(A)+S_{3}(A)\right]$, where $\phi(A)$ is a characteristic non-emptiness function of a set $A$, i. e. $\phi(A)=1$ if $A \notin \emptyset$ and $\phi(A)=0$ otherwise. $S_{1} \equiv m_{\mathcal{M}^{f}(\Theta)}, S_{2}(A)$, and $S_{3}(A)$ are defined for two sources (for n-ary versions see [13]) as it follows:

$S_{1}(A)=\sum_{X, Y \in D^{\Theta}, X \cap Y=A} m_{1}(X) m_{2}(Y)$, $S_{2}(A)=\sum_{X, Y \in \emptyset,[\mathcal{U}=A] \vee\left[(\mathcal{U} \in \emptyset) \wedge\left(A=I_{t}\right)\right]} m_{1}(X) m_{2}(Y)$,

$S_{3}(A)=\sum_{X, Y \in D^{\Theta}} \quad X \cup Y=A, X \cap Y \in \emptyset m_{1}(X) m_{2}(Y)$ with $\mathcal{U}=u(X) \cup u(Y)$, where $u(X)$ is the union of all singletons $\theta_{i}$ that compose $X$ and $Y$; all the sets $A, X, Y$ are supposed to be in some canonical form, e.g. CNF. Unfortunately no mention about the canonical form is included in [13]. $S_{1}(A)$ corresponds to the classic DSm rule on the free DSm model $\mathcal{M}^{f}(\Theta) ; S_{2}(A)$ represents the mass of all relatively and absolutely empty sets in both the input gbbas, which arises due to non-existential constraints and is transferred to the total or relative ignorance; and $S_{3}(A)$ transfers the sum of masses of relatively and absolutely empty sets, which arise as conflicts of the input gbbas, to the non-empty union of input sets.

The hybrid DSm rule generalizes the classic DSm rule to be applicable to any DSm model. The hybrid DSm rule is commutative but not associative. It is the reason the $\mathrm{n}$-ary version of the rule should be used in practical applications. For the n-ary version of $S_{i}(A)$, see [13].

\footnotetext{
${ }^{3} \emptyset$ should be $\emptyset_{\mathcal{M}}$ extended with the classic empty set $\emptyset$, thus more correct should be the expression $\emptyset=\emptyset_{\mathcal{M}} \cup\{\emptyset\}$.

${ }^{4}$ To distinguish the DSm rule from Dempster's rule, we use $\circledast$ instead of $\oplus$ for the DSm rule in this text.
} 


\section{A generalization of Dempster's rule}

Let us assume all elements $X$ from $D^{\Theta}$ to be in CNF in the rest of this contribution, unless another form of $X$ is explicitly specified. Let us also assume non-degenerated hybrid DSm models, i.e., $\Theta_{\mathcal{M}} \neq \emptyset, I_{\mathcal{M}} \notin \emptyset_{\mathcal{M}}$. Let us denote $\emptyset=\emptyset_{\mathcal{M}} \cup\{\emptyset\}$, i.e. set of set of all elements of $D^{\Theta}$ which are forced to be empty trough the constraints of DSm model $\mathcal{M}$ extended with classic empty set $\emptyset$, hence we can write $X \in \emptyset$ for all $X \stackrel{\mathcal{M}}{=} \emptyset$ including $\emptyset$.

The classic Dempster's rule puts belief mass $m_{1}(X) m_{2}(Y)$ to $X \cap Y$ (the rule adds it to $\left.\left(m_{1} \oplus m_{2}\right)(X \cap Y)\right)$ whenever it is non-empty, otherwise the mass is normalized. In the free DSm model all the intersections of non-empty elements are always non-empty, thus no normalization is necessary and Dempster's rule generalized to the free DSm model $\mathcal{M}^{f}(\Theta)$ coincides with the classic DSm rule: $\left(m_{1} \oplus m_{2}\right)(A)=$ $\sum_{X, Y \in D^{\Theta}, X \cap Y=A} m_{1}(X) m_{2}(Y)=\left(m_{1} \circledast m_{2}\right)(A)$.

Hence, Dempster's rule generalized to the free DSm model is defined for any couple of belief functions.

Empty intersections can appear in a general hybrid model due to the model's constraints, thus the normalization should be used.

The generalized Dempster's rule of combination $\oplus$ is given as

$$
\left(m_{1} \oplus m_{2}\right)(A)=\sum_{X, Y \in D^{\Theta}, X \cap Y \equiv A} K m_{1}(X) m_{2}(Y)
$$

for $\emptyset \neq A \in D_{\mathcal{M}}^{\Theta}$, where $K=\frac{1}{1-\kappa}, \kappa=\sum_{X, Y \in D^{\Theta}, X \cap Y \in \emptyset} m_{1}(X) m_{2}(Y)$, and $\left(m_{1} \oplus m_{2}\right)(A)=0$ otherwise, i.e., for $A=\emptyset$ and for $A \notin D_{\mathcal{M}}^{\Theta}$.

Similarly to the classic case, the generalized Demspter's rule is not defined in fully contradictive cases ${ }^{5}$ in hybrid DSm models, i.e. whenever $\kappa=1$. Specially the generalized Dempster's rule is not defined (and it cannot be defined) on the degenerated DSm model $\mathcal{M}_{\emptyset}$.

To be easily comparable with the DSm rule, we can rewrite the definition of the generalized Dempster's rule to the following equivalent form: $\left(m_{1} \oplus m_{2}\right)(A)=$ $\phi(A)\left[S_{1}^{\oplus}(A)+S_{2}^{\oplus}(A)+S_{3}^{\oplus}(A)\right]$, where $\phi(A)$ is a characteristic non-emptiness function of a set $A$, i. e. $\phi(A)=1$ if $A \notin \emptyset$ and $\phi(A)=0$ otherwise, $S_{1}^{\oplus}(A), S_{2}^{\oplus}(A)$, and $S_{3}^{\oplus}(A)$ are defined by

$S_{1}^{\oplus}(A)=S_{1}(A)=\sum_{X, Y \in D^{\Theta}, X \cap Y \equiv A} m_{1}(X) m_{2}(Y)$,

$S_{2}^{\oplus}(A)=\frac{S_{1}(A)}{\sum_{Z \in D^{\Theta}} Z \notin \emptyset S_{1}(Z)} \sum_{X, Y \in \emptyset_{\mathcal{M}} m_{1}(X) m_{2}(Y)}$,

$S_{3}^{\oplus}(A)=\frac{S_{1}(A)}{\sum_{Z \in D^{\Theta}} z \notin \emptyset S_{1}(Z)} \sum_{X, Y \in D^{\Theta}, X \cup Y \notin \emptyset, X \cap Y \in \emptyset_{\mathcal{M}}} m_{1}(X) m_{2}(Y)$.

\footnotetext{
${ }^{5}$ Note that in a static combination it means a full conflict/contradiction between input BFs. Whereas in the case of a dynamic combination it could be also a full conflict between mutually non-conflictling or partilally conflicting input BFs and constraints of a used hybrid DSm model. E.g. $m_{1}\left(\theta_{1} \cup \theta_{2}\right)=1, m_{2}\left(\theta_{2} \cup \theta_{3}\right)=$ 1 , where $\theta_{2}$ is constrained in a used hybrid model.
} 
$S_{1}^{\oplus}(A)$ corresponds to a non-conflicting belief mass, $S_{3}^{\oplus}(A)$ includes all classic conflicting masses and the cases where one of $X, Y$ is excluded by a non-existential constraint, and $S_{2}^{\oplus}(A)$ corresponds to the cases where both $X$ and $Y$ are excluded by (a) non-existential constraint(s).

It is easy verify that the generalized Dempster's rule coincides with the classic one on Shafer's model $\mathcal{M}^{0}$, for proof see [6]. Hence, the above definition of the generalized Dempster's rule is really a generalization of the classic Dempster's rule.

\section{A generalization of Yager's rule}

The classic Yager's rule puts belief mass $m_{1}(X) m_{2}(Y)$ to $X \cap Y$ whenever it is nonempty, otherwise the mass is added to $m(\Theta)$. As all the intersections are non-empty in the free DSm model, nothing should be added to $m_{1}(\Theta) m_{2}(\Theta)$ and Yager's rule generalized to the free DSm model $\mathcal{M}^{f}(\Theta)$ also coincides with the classic DSm rule. $\left(m_{1} \oslash m_{2}\right)(A)=\sum_{X, Y \in D^{\ominus}, X \cap Y=A} m_{1}(X) m_{2}(Y)=\left(m_{1} \circledast m_{2}\right)(A)$.

The generalized Yager's rule of combination (1) for a general hybrid DSm model $\mathcal{M}$ is given as

$$
\left(m_{1} \otimes m_{2}\right)(A)=\sum_{X, Y \in D^{\Theta}, X \cap Y \equiv A} m_{1}(X) m_{2}(Y)
$$

for $A \notin \emptyset, \Theta_{\mathcal{M}} \neq A \in D_{\mathcal{M}}^{\Theta}$,

$$
\left(m_{1} \otimes m_{2}\right)\left(\Theta_{\mathcal{M}}\right)=\sum_{\substack{X, Y \in D \Theta \\ X \cap Y \equiv \Theta_{\mathcal{M}}}} m_{1}(X) m_{2}(Y)+\sum_{\substack{X, Y \in D \Theta \\ X \cap Y \in \Phi_{\mathcal{M}}}} m_{1}(X) m_{2}(Y)
$$

and $\left(m_{1} \otimes m_{2}\right)(A)=0$ otherwise, i.e., for $A \in \emptyset$ and for $A \in\left(D^{\Theta} \backslash D_{\mathcal{M}}^{\Theta}\right)$.

To be easily comparable with the DSm rule, we can rewrite the definition of the generalized Yager's rule to an equivalent form: $\left(m_{1} \circledast m_{2}\right)(A)=\phi(A)\left[S_{1}^{\circledR}(A)+\right.$ $\left.S_{2}^{\bigotimes}(A)+S_{3}^{\bigotimes}(A)\right]$, where $S_{1}^{\bigotimes}(A), S_{2}^{\bigotimes}(A)$, and $S_{3}^{\bigotimes}(A)$ are defined by

$S_{1}^{\otimes}(A)=S_{1}(A)=\sum_{X, Y \in D^{\Theta}, X \cap Y \equiv A} m_{1}(X) m_{2}(Y)$,

$S_{2}^{\circledR}\left(\Theta_{\mathcal{M}}\right)=\sum_{X, Y \in \emptyset_{\mathcal{M}}} m_{1}(X) m_{2}(Y), S_{2}^{\circledR}(A)=0$ for $A \neq \Theta_{\mathcal{M}}$,

$S_{3}^{(囚)}\left(\Theta_{\mathcal{M}}\right)=\sum_{X, Y \in D^{\Theta}, X \cup Y \notin \emptyset, X \cap Y \in \emptyset_{\mathcal{M}}} m_{1}(X) m_{2}(Y), S_{3}^{(\otimes)}(A)=0$ for $A \neq \Theta_{\mathcal{M}}$.

It is easy to verify that the generalized Yager's rule coincides with the classic one on Shafer's model $\mathcal{M}^{0}$, for proof see [6]. Hence the definition of the generalized Yager's rule is really a generalization of the classic Yager's rule.

\section{A generalization of Dubois-Prade's rule}

The classic Dubois-Prade's rule puts belief mass $m_{1}(X) m_{2}(Y)$ to $X \cap Y$ whenever it is non-empty, otherwise the mass $m_{1}(X) m_{2}(Y)$ is added to $X \cup Y$ which is always 
non-empty in the DST.

In the free DSm model all the intersections of non-empty elements are always nonempty, thus nothing to be added to unions and Dubois-Prade's rule generalized to the free model $\mathcal{M}^{f}(\Theta)$ also coincides with the classic DSm rule.

$\left(m_{1} \circledast m_{2}\right)(A)=\sum_{X, Y \in D^{\Theta}, X \cap Y=A} m_{1}(X) m_{2}(Y)=\left(m_{1} \circledast m_{2}\right)(A)$.

In the case of a static fusion, only exclusivity constraints are used, thus all the unions of $X_{i} \in D^{\Theta}, X \notin \emptyset$ are also out of $\emptyset$. Thus we can easily generalize DuboisPrade's rule as $\left(m_{1} \circledast m_{2}\right)(A)=$

$\sum_{X, Y \in D^{\Theta}, X \cap Y=A} m_{1}(X) m_{2}(Y)+\sum_{X, Y \in D^{\Theta}, X \cap Y \in \emptyset_{\mathcal{M}}, X \cup Y=A} m_{1}(X) m_{2}(Y)$ for $A \in D^{\Theta}, A \notin \emptyset$, and $\left(m_{1} \circledast m_{2}\right)(A)=0$ for $A \in \emptyset$.

The situation is more complicated in the case of a dynamic fusion, where nonexistential constraints are used. There are several sub-cases how $X \cap Y \in \emptyset$ arises, for detail see [6].

Thus we can now formulate a definition of the generalized Dubois-Prade rule. We can distinguish three cases of input generalized belief functions: (i) all inputs satisfy all the constraints of a hybrid DSm model $\mathcal{M}(\Theta)$ which is used (a static belief combination), (ii) inputs do not satisfy the constraints of $\mathcal{M}(\Theta)$ (a dynamic belief combination), but no non-existential constraint is used, (iii) completely general inputs which do not satisfy the constraints, and non-existential constraints are allowed (a more general dynamic combination). According to these three cases, we can formulate three variants of the generalized Dubois-Prade rule.

The simple generalized Dubois-Prade rule of combination is given as ${ }^{6}$

$$
\left(m_{1} \circledast m_{2}\right)(A)=\sum_{X \cap Y \equiv A} m_{1}(X) m_{2}(Y)+\sum_{\substack{X \cap Y \in \emptyset_{\mathcal{M}} \\ X \cup Y \equiv A}} m_{1}(X) m_{2}(Y)
$$

for $\emptyset \neq A \in D_{\mathcal{M}}^{\Theta}$, and $\left(m_{1} \circledast m_{2}\right)(A)=0$ otherwise, i.e., for $A=\emptyset$ and for $A \in\left(D^{\Theta} \backslash D_{\mathcal{M}}^{\Theta}\right)$.

The generalized Dubois-Prade rule of combination 10 is given as

$\left(m_{1} \circledast m_{2}\right)(A)=\sum_{X \cap Y \equiv A} m_{1}(X) m_{2}(Y)+\sum_{\substack{X \cap Y \in \emptyset_{\mathcal{M}} \\ X \cup Y \equiv A}} m_{1}(X) m_{2}(Y)+\sum_{\substack{X \cup Y \in \emptyset_{\mathcal{M}} \\ U_{X} \cup Y \equiv A}} m_{1}(X) m_{2}(Y)$

for $\emptyset \neq A \in D_{\mathcal{M}}^{\Theta}$, and $\left(m_{1} \circledast m_{2}\right)(A)=0$ otherwise, i.e., for $A=\emptyset$ and for $A \in\left(D^{\Theta} \backslash D_{\mathcal{M}}^{\Theta}\right)$, where $U_{X \cup Y}$ is disjunctive normal form of $X \cup Y$ with all $\cap$ s substituted with $\cup$ s.

The extended generalized Dubois-Prade rule of combination $\circledast$ is given as

\footnotetext{
${ }^{6}$ We present here 3 variants of the generalized Dubois-Prade rule, formulas for all of them include several summations over $X, Y \in D^{\Theta}$, where $X, Y$ are more specified with other conditions. To simplify the formulas in order to increase their readability, we do not repeat the common condition $X, Y \in D^{\Theta}$ in sums in all the following formulas for the generalized Dubois-Prade rule.
} 


$$
\begin{aligned}
\left(m_{1} \circledast m_{2}\right)(A) & =\sum_{X \cap Y \equiv A} m_{1}(X) m_{2}(Y)+\sum_{\substack{X \cap Y \in \mathscr{\Phi}_{\mathcal{M}} \\
X \cup Y \equiv A}} m_{1}(X) m_{2}(Y) \\
& +\sum_{\substack{X \cup Y \in \emptyset_{\mathcal{M}} \\
U_{X \cup Y} \equiv A}} m_{1}(X) m_{2}(Y)
\end{aligned}
$$

for $\emptyset \neq A \neq \Theta_{\mathcal{M}}, A \in D_{\mathcal{M}}^{\Theta}$,

$$
\begin{aligned}
\left(m_{1} \circledast m_{2}\right)\left(\Theta_{\mathcal{M}}\right) & =\sum_{X \cap Y \equiv \Theta_{\mathcal{M}}} m_{1}(X) m_{2}(Y)+\sum_{\substack{X \cap Y \in \emptyset_{\mathcal{M}} \\
X \cup Y \equiv \Theta_{\mathcal{M}}}} m_{1}(X) m_{2}(Y) \\
& +\sum_{\substack{X \cup Y \in \Phi_{\mathcal{M}} \\
U_{X \cup Y} \equiv \Theta_{\mathcal{M}}}} m_{1}(X) m_{2}(Y)+\sum_{\underbrace{}_{X \cup Y} \in \emptyset_{\mathcal{M}}} m_{1}(X) m_{2}(Y),
\end{aligned}
$$

and $\left(m_{1} \circledast m_{2}\right)(A)=0$ otherwise, i.e., for $A \in \emptyset$ and for $A \in\left(D^{\Theta} \backslash D_{\mathcal{M}}^{\Theta}\right)$, where $U_{X \cup Y}$ is disjunctive normal form of $X \cup Y$ with all $\cap$ s substituted with $\cup$ s.

It is easy to verify that the generalized Dubois-Prade rule coincides with the classic one in Shafer's model $\mathcal{M}^{0}$, for proof see [6].

The classic Dubois-Prade rule is not associative, neither the generalized one is. Similary to the DSm approach we can easily rewrite the definitions of the (generalized) Dubois-Prade rule for a combination of $k$ sources.

To be easily comparable with the DSm rule, we can rewrite the definitions of the generalized Dubois-Prade rules to an equivalent form similar to that of DSm: the generalized Dubois-Prade rule:

$\left(m_{1} \circledast m_{2}\right)(A)=\phi(A)\left[S_{1}^{\circledast}(A)+S_{2}^{\circledast 0}(A)+S_{3}^{\circledast}(A)\right]$, where $S_{1}^{\circledast}(A)=S_{1}(A)=\sum_{X, Y \in D^{\ominus}, X \cap Y \equiv A} m_{1}(X) m_{2}(Y)$, $S_{2}^{\circledast 0}(A)=\sum_{X, Y \in \emptyset_{\mathcal{M}}, U_{X \cup Y} \equiv A} m_{1}(X) m_{2}(Y)$, $S_{3}^{\circledast 0}(A)=\sum_{X, Y \in D^{\Theta}, X \cap Y \in \emptyset_{\mathcal{M}},(X \cup Y) \equiv A} m_{1}(X) m_{2}(Y)$. the simple generalized Dubois-Prade rule: $\left(m_{1} \circledast m_{2}\right)(A)=\phi(A)\left[S_{1}^{\circledast}(A)+S_{3}^{\circledast}(A)\right]$, where $S_{1}^{\circledast}(A), S_{3}^{\circledast}(A)$ as above; the extended generalized Dubois-Prade rule: $\left(m_{1} \circledast m_{2}\right)(A)=\phi(A)\left[S_{1}^{\circledast}(A)+S_{2}^{\circledast}(A)+S_{3}^{\circledast}(A)\right]$, where $S_{1}^{\circledast}(A), S_{3}^{\circledast}(A)$ as above, and $S_{2}^{\otimes}(A)=\sum_{X, Y \in \emptyset_{\mathcal{M}},\left[U_{X \cup Y} \equiv A\right] \vee\left[U_{X \cup Y} \in \emptyset \wedge A=\Theta_{\mathcal{M}}\right]} m_{1}(X) m_{2}(Y)$.

For proofs see [6].

\section{A brief comparison of the rules}

As there are no conflicts in the free DSm model $\mathcal{M}^{f}(\Theta)$ all the presented rules coincide in the free DSm model $\mathcal{M}^{f}(\Theta)$. Thus the following statement holds: 
Statement 1 Dempster's rule, Yager's rule, Dubois-Prade's rule, the hybrid DSmH rule, and the classic DSmC rule are all mutually equivalent in the free DSm model $\mathcal{M}^{f}(\Theta)$.

Similarly the classic Dubois-Prade rule is equivalent to the DSm rule for Shafer's model. But in general all the generalized rules $\oplus,(1),(\rightarrow)$, and DSm rule are different. A very slight difference comes in the case of Dubois-Prade's rule and the DSm rule. A difference appears only in the case of a dynamic fusion, where some focal elements of both (of all in an n-ary case) the source basic belief assignments are equivalent to the empty set; an extension of the generalized Dubois-Prade rule is necessary there.

Statement 2 (i) If a hybrid DSm model $\mathcal{M}(\Theta)$ does not include any non-existential constraint or if all the input belief functions satisfy all the constraints of $\mathcal{M}(\Theta)$, then the generalized Dubois-Prade rule is equivalent to the DSm rule in the model $\mathcal{M}(\Theta)$.

(ii) The generalized Dubois-Prade rule extended with addition of $m_{1}(X) m_{2}(Y)$ (or $\Pi_{i} m_{i}\left(X_{i}\right)$ in an n-ary case) to $m(\Theta)$ for $X, Y \in \emptyset_{\mathcal{M}}$ (or for $X_{i} \in \emptyset_{\mathcal{M}}$ in an n-ary case) is fully equivalent to the hybrid DSmH rule on any hybrid DSm model.

For proofs see [6].

\subsection{Open problems}

As an open question remains commutativity of a transformation of generalized belief functions to those which satisfy all the constraints of a used hybrid DSm model with the particular combination rules. Such a commutation may significantly simplify functions $S_{2}$ and hence the entire definitions of the corresponding combination rules.

In the same way as it is used in this paper we can also generalize the non-normalized conjunctive rule of combination. A generalization of minC combination rule, whose computing mechanism (not a motivation nor an interpretation) has a relation to the conjunctive rules on the free DSm model $\mathcal{M}^{f}(\Theta)$ already in its classic case [3], is just under development [5].

We have to also mention the question of a possible generalization of conditionalization, related to particular combination rules to the domain of DSm hyper-power sets.

\section{Conclusion}

The classic rules for combination of belief functions have been generalized to be applicable to hyper-power sets, which are used in DSm theory. The generalization forms a solid theoretical background for full and objective comparison of the nature of the classic rules with the nature of the DSm rule of combination. It also enables us to place the DSmT better among the other approaches to belief functions. 


\section{Acknowledgement}

The support of the grant 1ET100300419 GA AV ČR is kindly announced.

The work was partly supported by the Institutional Research Plan AV0Z10300504

"Computer Science for the Information Society: Models, Algorithms, Applications".

\section{References}

[1] Cohen M. S. (1986), An expert system framework for non-monotonic reasoning about probabilistic assumptions, In Kanal, L.N., Lemmer, J.F. (eds.): Uncertainty in Artificial Intelligence 1. North-Holland.

[2] Daniel M. (2000), Distribution of Contradictive Belief Masses in Combination of Belief Functions. In: Bouchon-Meunier, B., Yager, R. R., Zadeh, L. A. (eds.): Information, Uncertainty and Fusion. Kluwer Academic Publishers 431-446.

[3] Daniel M. (2003), Associativity in Combination of belief functions; a derivation of minC combination. Soft Computing, 7/5 288-296.

[4] Daniel M. (2004), Algebraic Structures Related to the Combination of Belief Functions. Scientiae Mathematicae Japonicae, 60/2 245-255. Scientiae Mathematicae Japonicae Online, 10 501-511.

[5] Daniel M. (2006), A Generalization of the minC Combination to DSm Hyperpower Sets. Proceedings of IPMU 2006, (In Print).

[6] Daniel M. (2006), Classical Combination Rules Generalized to DSm Hyper-power Sets and their Comparison with the Hybrid DSm Rule. In: Smarandache F., Dezert J. (eds.): Advances and Applications of DSmT for Information Fusion, Vol.2, American Research Press, Rehoboth, (In Print).

[7] Dezert J. (2002), Foundations for a New Theory of Plausible and Paradoxical Reasoning. Information and Security Journal 9.

[8] Dubois D., Prade H. (1986), A Set-Theoretic View of Belief Functions. Int. J. General Systems, 12 193-226.

[9] Dubois D., Prade H. (1988), Representation an combination of uncertainty with belief functions and possibility measures. Computational Intelligence, 4244 264.

[10] Haenni R. (2005), Shedding New Light on Zadeh's Criticism of Dempster's Rule of Combination. In: Proceedings of Information Fusion 2005, Philadelphia, July 2005. 
[11] Lefevre E., Colot O., Vannoorenberghe P. (2002), Belief Functions Combination and Conflict Management. Information Fusion 3/2 149-162.

[12] Shafer G. (1976), A Mathematical Theory of Evidence. Princeton University Press, Princeton, New Jersey.

[13] Smarandache F., Dezert J. (2004), Advances and Applications of DSmT for Information Fusion. American Research Press, Rehoboth.

[14] Smets Ph. (1990), The combination of evidence in the transferable belief model. IEEE-Pattern analysis and Machine Intelligence, 12 447-458.

[15] Yager R. R. (1987), On the Demspter-Shafer framework and new combination rules. Information Sciences, 41 93-138.

MILAN DANIEL was born in Prague in 1962. He graduated in the Faculty of Mathematics and Physics of Charles University Prague in 1985. He defended his PhD thesis in the Institute of Computer Science of the Academy of Sciences of the Czech Republic in 1993. His research activities have been always related to the Institute, the department of Theoretical Computer Science, formerly the department of Knowledge Based Systems, see www.cs.cas.cz. Author's current main scientific interests are belief functions, namely combination of belief functions and probabilistic transformations of belief functions. The other interests are uncertainty processing, fuzzy logic and knowledge based systems. E-mail: milan.daniel@cs.cas.cz. 